\title{
Prolonged corticosteroid treatment in acute respiratory distress syndrome: impact on mortality and ventilator-free days
}

\author{
Gianfranco Umberto Meduri ${ }^{1 *}$, Bram Rochwerg ${ }^{2}$, Djillali Annane ${ }^{3}$, Stephen M. Pastores ${ }^{4}$ and on behalf of the \\ Corticosteroid Guideline Task Force of SCCM and ESICM
}

See related letter by Blot et al., https://ccforum.biomedcentral.com/articles/10.1186/s13054-017-1920-x

We read with interest the letter by Blot and colleagues recently published in Critical Care [1] which concluded that the recommendations of the Corticosteroid Guideline Task Force of SCCM and ESICM [2] for the use of adjunctive corticosteroids in early moderate to severe acute respiratory distress syndrome (ARDS) were based on insufficient evidence. In support of their view, the authors refer to the meta-analysis of Ruan et al. (reference [3] in Blot and colleagues' letter) also published in Critical Care in 2014. We respectfully disagree with their comments and offer the following observations. First, the meta-analysis by Ruan et al. did not take into account how current understanding of disease pathophysiology impacts the administration of corticosteroid treatment in ARDS. The meta-analysis by Ruan et al. incorporated four randomized trials from the 1980s that investigated short-term (24-48 h) massive daily corticosteroid doses (up to $120 \mathrm{mg} / \mathrm{kg}$ methylprednisolone equivalent), an intervention that is obsolete and discredited by the present pathophysiological understanding of ARDS [2]. Thus, the inclusion of these trials in the meta-analysis is mostly responsible for the inconsistency reported in their letter. Moreover, the conclusion by Ruan et al. that the benefits of corticosteroid treatment decreased over time are not supported by the actual findings of the cited trials (Figure 3 in [4], Figure 4 in [5] and Table 5 in [3]).

As the Corticosteroid Guideline Task Force state in the guideline, our recommendation for adjunctive use of corticosteroids in early moderate to severe ARDS is a conditional recommendation and not necessarily meant to imply a standard of care treatment. In our analysis [2] the pooled relative risk estimate for hospital mortality (Fig. 1)

\footnotetext{
* Correspondence: gmeduri@uthsc.edu

${ }^{1}$ Division of Pulmonary, Critical Care, and Sleep Medicine, Department of Medicine, Memphis Veterans Affairs Medical Center, (111) - 1030 Jefferson Avenue Suite room \#CW444 -, Memphis, TN 38104, USA

Full list of author information is available at the end of the article
}

with corticosteroids was 0.64 (95\% confidence interval (CI) 0.46-0.89). Even if one excludes the four studies which Blot and colleagues appear to question [1], the pooled relative risk estimate for hospital mortality is 0.76 (95\% CI 0.58-0. 99), which is not significantly different from 0.64 (0.46-0. 89). Independent of hospital mortality, the use of corticosteroids was associated with approximately a 7-day increase in ventilator-free days (mean difference 7.06 days, 95\% CI 3.19-10.93) [2] (supplementary digital content in [5]). Given that our recommendation for adjunctive corticosteroids in ARDS is conditional in nature, ongoing and future prospective trials will certainly impact our future recommendations.

\section{Funding}

No financial support was received. This letter is the result of work supported with the resources and use of facilities at the Memphis VA Medical Center. The contents of this letter do not represent the views of the US Department of Veterans Affairs or the United States Government.

\section{Authors' contributions}

GUM wrote the first draft and BR, SMP and DA reviewed, edited, and approved the final manuscript. All authors read and approved the final manuscript.

\section{Competing interests}

The authors declare that they have no competing interests.

\section{Publisher's Note}

Springer Nature remains neutral with regard to jurisdictional claims in published maps and institutional affiliations.
Author details
'Division of Pulmonary, Critical Care, and Sleep Medicine, Department of Medicine, Memphis Veterans Affairs Medical Center, (111) - 1030 Jefferson Avenue Suite room \#CW444 -, Memphis, TN 38104, USA. ${ }^{2}$ Division of Critical Care, Department of Medicine, McMaster University, Hamilton, ON, Canada. ${ }^{3}$ General ICU Department, Raymond Poincaré hospital (APHP), Health Science Centre Simone Veil, Université Versailles SQY-Paris Saclay, Paris, France.
${ }^{4}$ Department of Anesthesiology and Critical Care Medicine, Memorial Sloan Kettering Cancer Center, New York, NY, USA. 


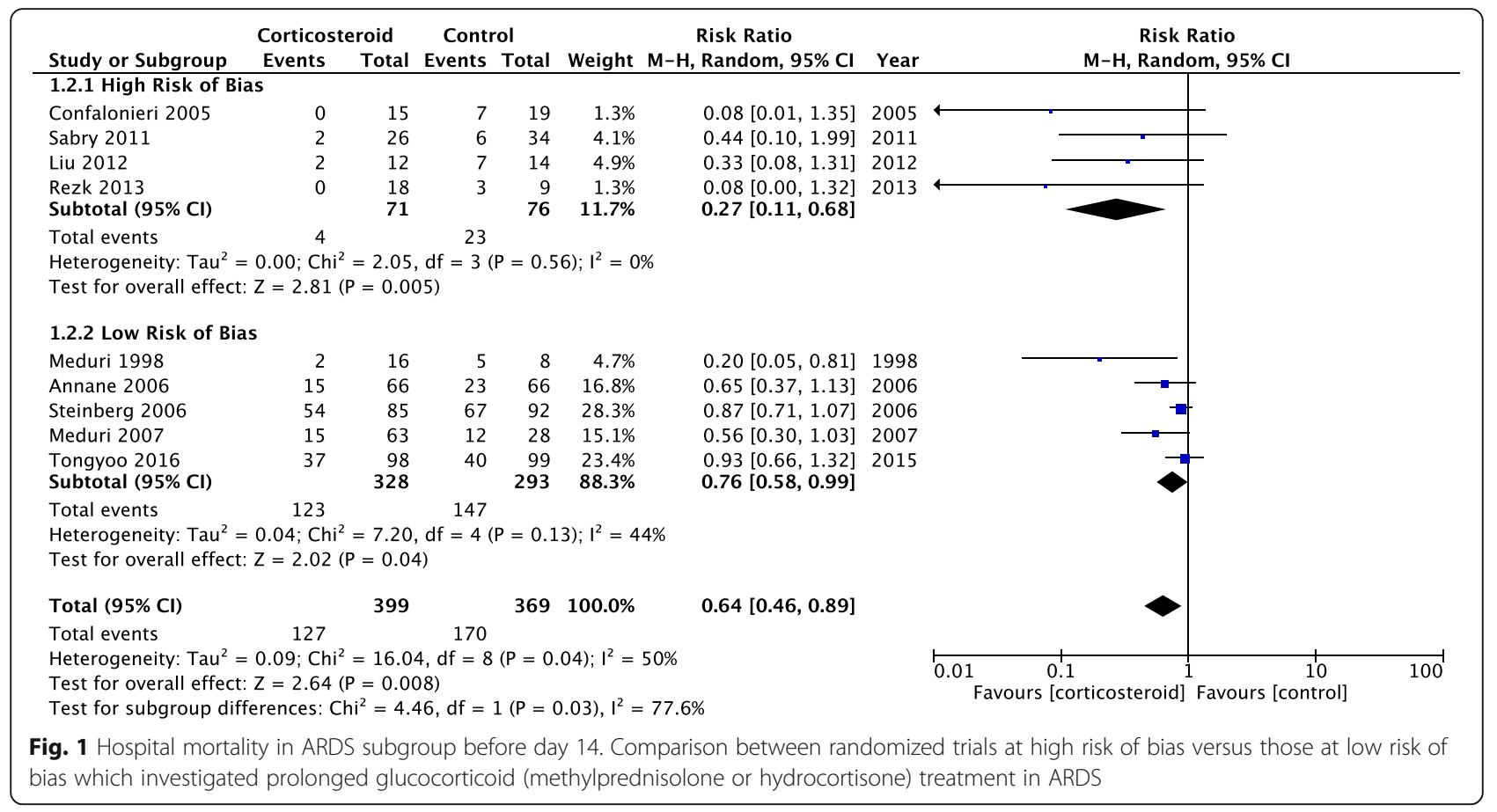

Received: 9 February 2018 Accepted: 25 February 2018

Published online: 24 May 2018

\section{References}

1. Blot M, Salmon-Rousseau A, Chavanet P, Piroth L. Do we know enough to recommend corticosteroids in acute respiratory distress syndrome? Crit Care. 2017;21(1):327.

2. Annane D, Pastores SM, Rochwerg B, et al. Guidelines to the diagnosis and management of critical illness-related corticosteroid insufficiency $(\mathrm{CIRCI})$ in critically ill patients (part 1): Society of Critical Care Medicine (SCCM) and European Society of Intensive Care Medicine (ESICM) 2017. Crit Care Med. 2017:45:2078-88

3. Annane D, Sebille V, Bellissant E. Ger-Inf-05 Study G: Effect of low doses of corticosteroids in septic shock patients with or without early acute respiratory distress syndrome. Crit Care Med. 2006;34(1):22-30.

4. Meduri GU, Headley AS, Golden E, Carson SJ, Umberger RA, Kelso T, Tolley EA. Effect of prolonged methylprednisolone therapy in unresolving acute respiratory distress syndrome: a randomized controlled trial. JAMA. 1998; 280(2):159-65

5. Meduri GU, Golden E, Freire AX, Taylor E, Zaman M, Carson SJ, Gibson M, Umberger R. Methylprednisolone infusion in early severe ARDS: results of a randomized controlled trial. Chest. 2007;131(4):954-63. 